\title{
Особенности кинетики сорбции низших алифатических альдегидов полифункциональным низкоосновным анионообменником
}

\author{
(С2020 Воронюк И.В., Елисеева Т.В., Селеменев В.Ф. \\ Воронежский государственный университет, Воронеж
}

Поступила в редакцию 19.11.2020 г.

DOI: $10.17308 /$ sorpchrom.2020.20/3145

Низшие алифатические альдегиды относятся к органическим экотоксикантам, негативно влияющим на состояние окружающей среды и здоровье человека. Извлечение таких соединений из водных сред - актуальная и практически важная задача, одним из способов решения которой является применение сорбционного метода с использованием в качестве сорбентов низкоосновных анионообменников. Ионообменные материалы с первичными, вторичными и третичными аминогруппами способны к поглощению карбонильных соединений за счет хемосорбционного взаимодействия.

Целью исследования являлось установление кинетических особенностей сорбции ряда алифатических альдегидов (метаналя, этаналя, пропаналя) низкоосновным анионообменником Purolite A 830 из модельных водных растворов в статических условиях. На основе полученных данных отмечено, что при высокой селективности применяемого сорбента к исследуемым сорбтивам время достижения равновесия для метаналя в несколько раз ниже, чем для этаналя и пропаналя $(24,195$ и 125 часов, соответственно). Подобное явление становится понятным при детальном рассмотрении химизма исследуемого процесса. В отличие от метаналя, альдегиды с большей длиной углеводородной цепи способны к межмолекулярным взаимодействиям в растворе - альдольной конденсации. При этом продукты такого взаимодействия также могут участвовать в сорбционном поглощении анионообменником. Возможно, что протекание побочных реакций оказывает существенное влияние на скорость сорбционного процесса, повышая при этом емкость по альдегиду.

Для описания полученных кинетических зависимостей в работе применяли формальный подход, заключающийся в выборе уравнений сорбции, максимально близко описывающих экспериментальные данные. В качестве моделей кинетики сорбции выбраны модели, используемые для описания адсорбционных процессов: уравнение псевдо-первого порядка, уравнение псевдо-второго порядка, уравнение Вебера-Морриса и уравнение Еловича. Линеаризация данных эксперимента в координатах этих уравнений позволила по величинам коэффициентов достоверности $\left(\mathrm{R}^{2}\right)$ подобрать модель кинетики сорбции, наиболее близко описывающую сорбцию низших алифатических альдегидов анионообменником Purolite A 830 во времени. Отмечено, что уравнение Еловича, разработанное для описания кинетики адсорбции в гетерогенных системах, применимо для описания изменения концентрации сорбтива в растворе при контакте с исследуемым полифункциональным низкоосновным анионообменником.

Ключевые слова: хемосорбция, кинетика, уравнение Еловича, формальная кинетика, алифатические альдегиды, низкоосновный анионообменник

\section{Введение}

Решение проблемы загрязнения окружающей среды с каждым годом все более актуально. Присутствие токсикантов в воде, почве и воздухе ухудшает качество жизни и приводит к пролонгированным негативным последствиям для экосферы. Наличие в сточных водах таких экотоксикантов, как метаналь (формальдегид), эта- 
наль (ацетальдегид) и пропаналь (пропионовый альдегид), связано с работой производств химической промышленности и мебельных предприятий. Попадание данных соединений в воды различного назначения чрезвычайно опасно, так как они относятся к канцерогенам [1].

Одним из перспективных методов выделения карбонильных соединений из жидких сред является сорбционный с использованием сорбентов как неорганической природы [2-6], так и полимерных анионообменников [7-8].

Цель настоящей работы - установление особенностей кинетики сорбции ряда алифатических альдегидов низкоосновным анионообменником Purolite A 830 в статических условиях.

\section{Экспериментальная часть}

В качестве растворов сорбтива использованы водные растворы метаналя, приготовленные путем разбавления из 36\%-го раствора формалина, растворы этаналя и пропаналя, приготовленные путем разбавления из 99\%-ых растворов соответствующих альдегидов (Panreac). Концентрацию метаналя определяли методом иодометрического титрования [9], концентрацию этаналя устанавливали методом фотометрии с использованием нитропруссида натрия [10], содержание пропаналя в растворе определяли методом фотометрии с использованием пирогаллола в сернокислой среде [10]. Исходная концентрация альдегидов в модельных растворах составляла $1.4 \cdot 10^{-2}$ моль/дм ${ }^{3}$.

Исследование особенностей извлечения представителей карбонильных соединений низкоосновным анионообменником проводилось с использованием в качестве сорбента макропористого анионообменника Purolite A 830, имеющего в своей структуре первичные, вторичные и третичные функциональные аминогруппы. Отношение объема раствора сорбтива к массе ионообменника в экспериментах составляло 200:1, перемешивание раствора осуществлялось на орбитальном шейкере. Достижение равновесного состояния в исследуемой системе оценивали путем анализа изменения концентрации сорбтива в растворе в результате контакта с выбранным анионообменником. Кинетические кривые сорбции приводили в координатах $\mathrm{F}-\tau$, где $\mathrm{F}=\mathrm{Q}_{\tau} / \mathrm{Q}_{\mathrm{e}}$ - степень завершенности процесса поглощения альдегида низкоосновным сорбентом; $\mathrm{Q}_{\tau}-$ емкость сорбента по альдегиду в момент времени $\tau$, ммоль $/ \Gamma ; \mathrm{Q}_{\mathrm{e}}$ - емкость сорбента по альдегиду в состоянии равновесия, ммоль/г; $\tau$ - время контакта сорбента с раствором сорбтива, мин.

\section{Обсуждение результатов}

В работе [11] подтвержден факт хемосорбционного взаимодействия альдегидов с функциональными группами низкоосновных анионообмеников. Основной реакцией является образование в матрице сорбента оснований Шиффа. Кроме того, при высоких концентрациях карбонильного соединения возможно протекание и других взаимодействий: для метаналя - образование триазиновых циклов, для этаналя альдольная конденсация с последующей сорбцией продуктов данной реакции.

Очевидно, что различие в природе взаимодействий метаналя и последующих низших альдегидов с анионообменником должны оказывать влияние и на сорбционные характеристики процесса. На рис. 1 приведены кинетические кривые сорбции ряда низших альдегидов Purolite A 830. 


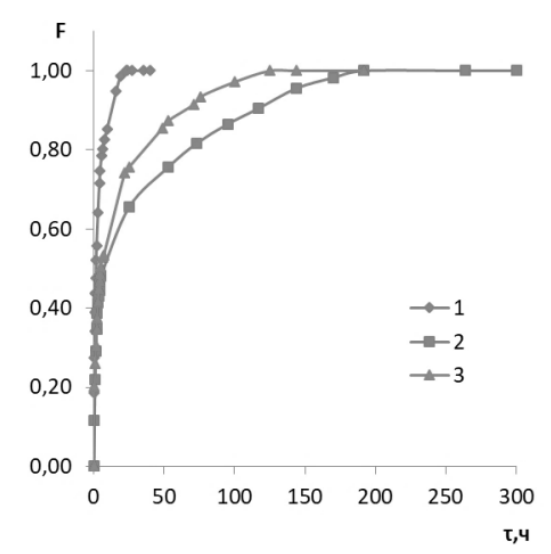

Рис. 1. Кинетические кривые сорбции алифатических альдегидов анионообменником Purolite A 830: 1 - метаналь, 2 - этаналь, 3 - пропаналь

Fig. 1. Kinetic curves of sorption of aliphatic aldehydes by the anion exchanger Purolite A 830: 1 - methanal, 2 - ethanal, 3 - propanal

Исследование сорбции альдегидов из водных растворов позволило определить емкость анионообменника по альдегидам и время, необходимое для достижения равновесного состояния в исследуемой системе (табл. 1).

Таблица 1. Сорбционные характеристики анионообменника Purolite A 830 при извлечении алифатических альдегидов из водных растворов

Table 1. Sorption characteristics of the Purolite A 830 anion exchanger during the extraction of aliphatic aldehydes from aqueous solutions

\begin{tabular}{|c|c|c|c|}
\hline \multirow{2}{*}{ Параметр } & \multicolumn{3}{|c|}{ Алифатический альдегид } \\
\cline { 2 - 4 } & Метаналь & Этаналь & Пропаналь \\
\hline $\begin{array}{c}\text { Емкость } \\
\text { по альдегиду, } \\
\text { ммоль/ }\end{array}$ & 3.1 & 4.5 & 4.9 \\
\hline $\begin{array}{c}\text { Время достижения } \\
\text { равновесия, ч }\end{array}$ & 24 & 195 & 125 \\
\hline
\end{tabular}

Экстремально низкая скорость сорбционного процесса характерна для альдегидов с углеводородной цепью С2-С3 и связана с процессом их альдольной конденсации в матрице анионообменника, катализируемой функциональными группами. При этом можно предположить, что продукты конденсации альдегидов будут также закрепляться в матрице за счет хемосорбционного взаимодействия с образованием непредельных иминов.

Отличие во времени достижения равновесия между системами с этаналем и пропаналем связано с тем, что с ростом углеводородной цепи реакция альдольной конденсации идет легче [12], что ускоряет сорбционный процесс.

Установление лимитирующей стадии сорбционного процесса проводили с применением формального подхода, основанного на применении известных уравнениях сорбции [13-16], позволяющих в первом приближении оценить роль диффузии и самой химической реакции в процессе сорбционного извлечения альдегидов. Такой подход широко используется для анализа кинетики сорбции органических веществ сорбентами различной природы [17-20].

В общем случае хемосорбционный процесс поглощения алифатических альдегидов можно представить как гетерогенную реакцию, основанную на взаимодей- 
ствии реакционноспособных групп анионообменника $\overline{R X}$ (адсорбционных центров) с молекулой сорбтива А:

$$
\overline{R X}+A \leftrightarrow \overline{R X A} .
$$

Скорость поглощения вещества сорбентом рассчитывается как изменение количества поглощенного вещества за единицу времени:

$$
\vartheta=\frac{d Q}{d \tau}
$$

где $d Q=Q_{e}-Q_{\tau}$ - количество поглощенного сорбтива за промежуток времени $d \tau ; Q_{e}$ и $Q_{\tau}$ - емкости сорбента в состоянии равновесия и в момент времени $\tau$, соответственно, ммоль/г.

При этом представление экспериментальных данных в координатах интегральных форм кинетических уравнений псевдо-первого порядка (1), псевдо-второго порядка (2), уравнения Вебера-Морриса (3) и уравнения Еловича (4) позволяет по величине коэффициента достоверности линейной зависимости выбрать наиболее подходящее математическое выражение.

$$
\begin{gathered}
\lg \left(Q_{e}-Q_{\tau}\right)=\lg Q_{e}-\frac{k_{p 1}}{2.303} \tau, \\
\frac{\tau}{Q_{\tau}}=\frac{1}{k_{p 2} \cdot Q_{e}^{2}}+\frac{1}{Q_{e}} \tau, \\
Q_{\tau}=k_{i d} \cdot \tau^{0.5}+c, \\
Q_{\tau}=\frac{1}{\beta} \ln (\alpha \beta)+\frac{1}{\beta} \ln \tau,
\end{gathered}
$$

где $\mathrm{k}_{\mathrm{p} 1}$ - константа скорости сорбции модели псевдо-первого порядка, $\mathrm{c}^{-1} ; \mathrm{k}_{\mathrm{p} 2}$ - константа скорости сорбции модели псевдо-второго порядка, г/(ммоль $\cdot c) ; \mathrm{k}_{\mathrm{id}}-$ константа скорости внутренней диффузии, ммоль $/\left(\Gamma^{\cdot} \mathrm{c}^{0.5}\right) ; \mathrm{c}-$ параметр, характеризующий влияние пограничного слоя на скорость сорбции; $\alpha$ - начальная скорость сорбционного процесса, ммоль/(г·c); $\beta$ - константа десорбции, г/ммоль.

Все используемые в работе кинетические модели сорбции позволяют оценить вклад в сорбцию скорости акта взаимодействия между сорбентом и сорбтивом. При этом модель Вебера-Морриса учитывает и влияние скорости внутренней диффузии молекул альдегида в матрице анионообменника. На рис. 2 представлены кинетические кривые сорбции метаналя, этаналя и пропаналя исследуемым сорбентом в координатах уравнений (1)-(4).

В таблице 2 представлены результаты линеаризации кинетических кривых сорбции в координатах уравнений псевдо-первого, псевдо-второго порядков и урав-

\begin{tabular}{|c|c|c|c|c|c|c|}
\hline & \multicolumn{6}{|c|}{ Кинетические модели сорбции } \\
\hline & \multicolumn{2}{|c|}{$\begin{array}{l}\text { псевдо- } \\
\text { первого } \\
\text { порядка }\end{array}$} & \multicolumn{2}{|c|}{$\begin{array}{c}\text { псевдо-второго } \\
\text { порядка }\end{array}$} & \multicolumn{2}{|c|}{$\begin{array}{c}\text { модифицированная } \\
\text { модель внутренней диффузии } \\
\text { Вебера-Морриса }\end{array}$} \\
\hline & $\mathrm{R}^{2}$ & $\mathrm{k}_{\mathrm{p} 1, \mathrm{c}^{-1}}$ & $\mathrm{R}^{2}$ & $\begin{array}{c}\mathrm{k}_{\mathrm{p} 2} \\
\Gamma \cdot(\text { Ммоль } \cdot \mathrm{c})^{-1}\end{array}$ & $\mathrm{R}^{2}$ & 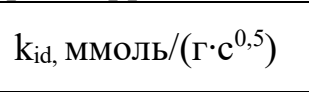 \\
\hline Метаналь & 0.972 & $5 \cdot 10^{-5}$ & 0.957 & $7 \cdot 10^{-5}$ & 0.898 & $1 \cdot 10^{-2}$ \\
\hline Этаналь & 0.974 & $5 \cdot 10^{-6}$ & 0.961 & $9 \cdot 10^{-6}$ & 0.925 & $4 \cdot 10^{-3}$ \\
\hline Пропаналь & 0.983 & $9 \cdot 10^{-6}$ & 0.933 & $1 \cdot 10^{-5}$ & 0.934 & $6 \cdot 10^{-3}$ \\
\hline
\end{tabular}
нения Вебера-Морриса.

Таблица 2. Результаты обработки кинетических кривых сорбции альдегидов анионообменником А 830 согласно различным моделям химической кинетики

Table 2. Results of processing kinetic curves of aldehyde sorption by anion exchanger A 830 according to various models of chemical kinetics 
Однако наиболее подходящим уравнением кинетики для описания закономерностей сорбции алифатических альдегидов анионообменником является уравнение Еловича, используемое для случая кинетики адсорбции в гетерогенных системах с учетом их сорбционной емкости (табл. 3)

Таблица 3. Кинетические характеристики сорбции карбонильных соединений низкоосновным анионообменником Purolite A 830

Table 3. Kinetic characteristics of sorption of carbonyl compounds by weakly basic anion exchanger Purolite A 830

\begin{tabular}{|c|c|c|c|}
\hline & $\mathrm{R}^{2}$ & $\alpha, \mathrm{ммоль} /(\Gamma \cdot \mathrm{c})$ & $\beta, \Gamma /$ ммоль \\
\hline Метаналь & 0.991 & $2 \cdot 10^{-3}$ & 1.4 \\
\hline Этаналь & 0.994 & $1 \cdot 10^{-3}$ & 1.6 \\
\hline Пропаналь & 0.996 & $1 \cdot 10^{-3}$ & 1.3 \\
\hline
\end{tabular}
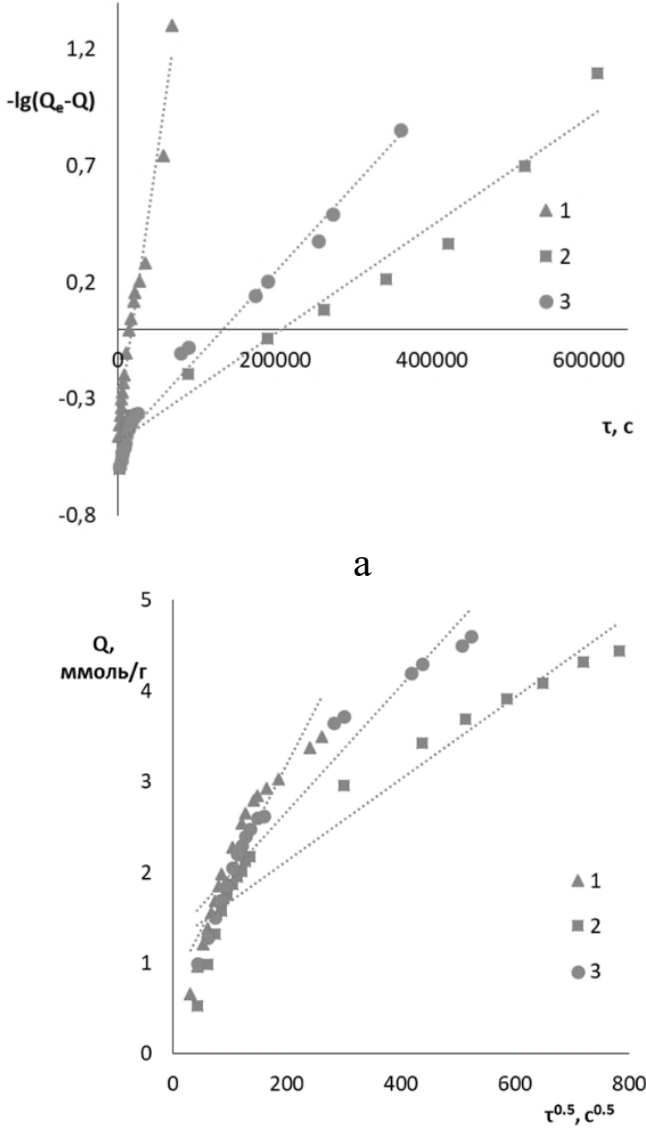

B

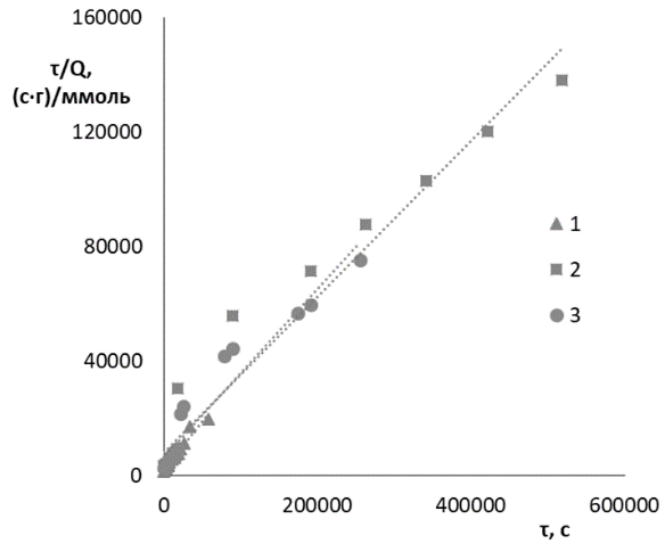

6

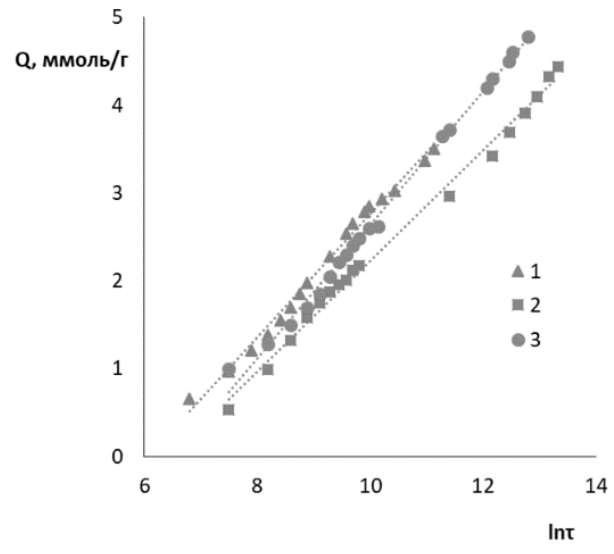

$\Gamma$

Рис. 2. Кинетические кривые сорбции низших алифатических альдегидов

из водных растворов анионообменником Purolite A 830, представленные

в координатах уравнения псевдо-первого порядка (а), псевдо-второго порядка (б), внутренней диффузии (в), Еловича (г): 1 - раствор метаналя, 2 - раствор этаналя, 3 - раствор пропаналя.

Fig. 2. Kinetic curves of the sorption of lower aliphatic aldehydes from aqueous solutions by the Purolite A 830 anion exchanger, presented in the coordinates of the pseudofirst order (a), pseudo-second order (b), internal diffusion (c), Elovich (d) equations. 1 - methanal solution, 2 - ethanal solution, 3 - propanal solution.

Исходя из специфики хемосорбционного взаимодействия в системе анионообменник - раствор низшего алифатического альдегида, можно предположить, что 
на скорость достижения равновесного состояния будут оказывать влияние не только диффузионные процессы, но и скорость взаимодействия сорбтива с функциональными группами сорбента. При этом поверхность анионообменника можно представлять как гетерогенную, где адсорбционными центрами являются реакционноспособные функциональные аминогруппы.

\section{Заключение}

Изучение кинетики сорбции алифатических альдегидов из водных растворов с концентрацией $4 \cdot 10^{-2}$ моль/дм ${ }^{3}$ низкоосновным анионообменником Purolite A 830 указывает на некоторые особенности исследуемого процесса. Емкостные характеристики свидетельствуют о селективности используемого сорбента для извлечения карбонильных соединений из водных сред, однако следует учитывать длительность достижения равновесного состояния в системе, особенно в случае сорбции этаналя и пропаналя.

Сложный многовариантный хемосорбционный процесс поглощения альдегидов с углеводородной цепью С2-С3 требует учета влияния на скорость сорбционного процесса не только стадий внешней и внутренней диффузии сорбтива, но и скорости протекания самого акта химического взаимодействия альдегида с функциональными группами сорбента, а также межмолекулярных взаимодействий сорбтив-сорбтив в растворе с последующей сорбцией продуктов их реакции (процесс альдольной конденсации с образованием непредельных альдегидов).

При исследовании кинетики сорбции низших алифатических альдегидов анионообменными материалами необходимо рассматривать получаемые результаты не только с позиции традиционных для ионообменных систем диффузионных кинетических моделей, но и моделей кинетики адсорбции, учитывающих влияние скорости акта взаимодействия сорбтив-сорбент. Отмечено, что для описания процесса взаимодействия альдегидов с анионообменником Purolite A 830 лучше всего подходит модель Еловича, учитывающая гетерогенность сорбента при извлечении сорбтива из раствора.

\section{Работа выполнена при поддержке Министерства науки и высшего образования РФ в рамках государственного задания ВУЗам в сфере научной деятельности на 2020-2022 годbl, проект № FZGU-2020-0044»}

\section{Список литературы}

1. СанПиН 1.2.2353-08 Канцерогенные факторы и основные требования к профилактике канцерогенной опасности.

2. Бельчинская Л.И., Ткачева О.А., Ермолов Ю.В. // Актуальные направления научных исследований XXI века: теория и практика. 2014. № 2-1. С.359-363.

3. Novikova L.A., Bogdanov D.S., Belchinskaya L.I., Kolousek D. et al. // Protection of Metals and Physical Chemistry of Surfaces. 2019. Vol.55. pp. 864-871.

4. Zvulunov Yu., Ben-Barak-Zelas Z., Fishman A., Radian A. // Chemical Engineering Journal. 2019. Vol. 374. pp. 1275-1285.
5. ManMan Wang, BaoMin Fan, BianYing Wen, Chao Jiang // Science China Technological Sciences. 2020. Vol. 63 pp. 2098-2112.

6. Zahed M., Jafari D., Esfandyari M. // International Journal of Environmental Analytical Chemistry. 2020. pp. 1-23. DOI: 10.1080/03067319.2020.1762872

7. Ghumro T., Junejo B., Solangi A.R., Memon S.Q. // Pak. J. Anal. Environ. Chem. 2019. Vol. 20. No 2. pp. 161-168.

8. Воронюк И.В., Елисеева Т.В., Селеменев В.Ф. // Журнал физической химии. 2012. T. 86. № 5. C. 984-986. 
9. Массовая концентрация формальдегида в водах. Методика выполнения измерений фотометрическим методом с ацетилацетоном : РД 52.24.492-2006. Ростов н/Д. 2006.

10.Коренман И.М. Фотометрический анализ. Методы определения органических соединений. М. Химия. 1970. 342 с.

11. Воронюк И.В., Елисеева Т.В. // Высокомолекулярные соединения. 2015. Т. 57. № 1. C. 55-61.

12.Агрономов А.Е. Избранные главы органической химии. М. Химия. 1990. 560 с.

13.Lagergren S., Sven K. // Vetenskapsakad. Handl. 1898. Vol. 24. No 4. pp. 1-39.

14.Ho Y.S., McKay G. // Process Biochem. 1999. Vol. 34. pp. 451-465.

15.Weber J.G., Asce J.M., Morris J.C. // J. Sanit Eng Div Am Soc Civ Engrs. 1963. Vol. 89. pp. 31-59.
16.Ho Y.S., Ng J.C.Y., McKay G. // Separ. Purif. Methods. 2000. Vol. 29. No 2. pp. 189232.

17.Крижановская О.О., Синяева Л.А., Карпов С.И., Селеменев В.Ф. и др. // Сорбиионные и хроматографические проиессы. 2014. T. 14. № 5. C. 784-794.

18.Корж Е.А., Клименко Н.А. // Проблемы современной науки и образования. 2017. Т. 87. № 5. C. 7-13.

19.Suteu D., Coseri S., Rusu L. // Desalination and Water Treatment. 2015. Vol. 57. No $31 . \quad$ pp. 14665-14673. doi:10.1080/19443994.2015.1063464

20. Yousef R., Malika C. // Water Sci Technol. 2020. Vol. 82. No 11. pp. 2366-2380. doi:10.2166/wst.2020.505

\title{
Features of the kinetics of lower aliphatic aldehydes sorption by a polyfunctional weakly basic anion exchanger
}

\author{
(C) 2020 Voronyuk I.V., Eliseeva T.V., Selemenev V.F. \\ Voronezh State University, Voronezh
}

\begin{abstract}
Carbonyl compounds are organic pollutants that negatively affect the environment and human health. The extraction of such compounds from liquid media is an urgent and practically important problem, which can be solved by the sorption method using weakly basic anion exchangers as sorbents. Ion exchange materials with primary, secondary, and tertiary amino groups are capable of absorbing carbonyl compounds due to chemisorption interaction.

The aim of the study was establishment of the features of the sorption of a number of aliphatic aldehydes (methanal, ethanal, propanal) by the weakly basic anion exchanger Purolite A 830 from model aqueous solutions under static conditions. Based on obtained data, it was noted that, with a high selectivity of the applied sorbent to the studied sorbtives, the time of achieving equilibrium for methanal was several times lower than for ethanal and propanal $(24,195$, and 125 hours, respectively). This phenomenon becomes understandable based on the result of a detailed examination of the chemistry of the studied process. Unlike methanal, ethanal and propanal are capable of intermolecular interactions in a solution, i.e. aldol condensation. In this case the products of such interactions can be also absorbed by the anion exchanger. It is possible that the occurrence of side reactions has a significant effect on the rate of the sorption process, increasing the sorbent capacity to aldehyde.

For the interpretation obtained kinetic dependences, a formal approach, consisting in choosing the sorption equations describing the experimental data as accurately as possible was used in this study. The models used for the description of the adsorption processes were selected as models for the kinetics of sorption: the pseudo-first order equation, the pseudo-second order equation, the Weber-Morris equation and the Elovich equation. The linearisation of the experimental data in the coordinates of these equations and comparison of the reliability coefficients values $\left(\mathrm{R}^{2}\right)$ allowed choosing a model of the sorption kinetics most closely describing the sorption of the lower aldehydes by Purolite A 830 in time. It was noted that the use of the Elovich equation, which was developed to describe the kinetics of adsorption in heterogeneous systems, is applicable for the description of the change in the concentration of a sorbtive in a solution upon contact with a weakly basic anion exchanger.
\end{abstract} exchanger.

Keywords: chemisorption, kinetics, Elovich equation, formal kinetics, aliphatic aldehydes, anion 


\section{References}

1. SanPiN 1.2.2353-08 Kancerogennye faktory i osnovnye trebovaniya $\mathrm{k}$ profilaktike kancerogennoj opasnosti.

2. Bel'chinskaya L.I., Tkacheva O.A., Ermolov YU.V., Aktual'nye napravleniya nauchnyh issledovanij XXI veka: teoriya i praktika. 2014. № 2-1. S.359-363.

3. Novikova L.A., Bogdanov D.S., Belchinskaya L.I., Kolousek D. et al., Protection of Metals and Physical Chemistry of Surfaces, 2019, Vol. 55, pp. 864-871.

4. Zvulunov Yu., Ben-Barak-Zelas Z., Fishman A., Radian A., Chemical Engineering Journal, 2019, Vol. 374, pp. 1275-1285.

5. ManMan Wang, BaoMin Fan, BianYing Wen, Chao Jiang, Science China Technological Sciences, 2020, Vol. 63, pp. 2098-2112.

6. Zahed M., Jafari D., Esfandyari M., International Journal of Environmental Analytical Chemistry, 2020, pp. 1-23. DOI: 10.1080/03067319.2020.1762872

7. Ghumro T., Junejo B., Solangi A.R., Memon S.Q., Pak. J. Anal. Environ. Chem., 2019, Vol. 20, No 2, pp. 161-168.

8. Voronyuk I.V., Eliseeva T.V., Selemenev V.F., Zhurnal fizicheskoj khimii, 2012, Vol. 86, No 5, pp. 984-986.

9. Massovaya koncentraciya formal'degida $\mathrm{v}$ vodah. Metodika vypolneniya izmere-nij fotometricheskim metodom $\mathrm{s}$ acetilacetonom : RD 52.24.492-2006. Rostov n/D. 2006.

Воронюк Ираида Владимировна - к.Х.н., доцент кафедры аналитической химии ФГБОУ ВО «Воронежский государственный университет», Воронеж

Елисеева Татьяна Викторовна - к.Х.н., зав. кафедрой аналитической химии ФГБОУ ВО «Воронежский государственный университет», Воронеж

Селеменев Владимир Федорович - д.Х.н., проф. каф. аналитической химии, Воронежский государственный университет, Воронеж
10.Korenman I.M. Fotometricheskij analiz. Metody opredeleniya organicheskih soedinenij. M. Khimiya, 1970, 342 p.

11. Voronyuk I.V., Eliseeva T.V., Vysokomolekulyarnye soedineniya, 2015, Vol. 5, No 1, pp. 55-61.

12.Agronomov A.E., Izbrannye glavy organicheskoj khimii, M., Khimiya, 1990, 560 p.

13.Lagergren S., Sven K., Vetenskapsakad. Handl., 1898, Vol. 24, No 4, pp. 1-39.

14.Ho Y.S., McKay G., Process Biochem., 1999, Vol. 34, pp. 451-465.

15.Weber J.G., Asce J.M., Morris J.C., J. Sanit. Eng. Div. Am. Soc. Civ. Engrs., 1963, Vol. 89, pp. 31-59.

16.Ho Y.S., Ng J.C.Y., McKay G., Separ. Purif. Methods., 2000. Vol. 29, No 2, pp. 189-232.

17.Krizhanovskaya O.O., Sinyaeva L.A., Karpov S.I., Selemenev V.F. et al., Sorbtsionnye i khromatograficheskie protsessy, 2014, Vol. 14, No 5, pp. 784-794.

18.Korzh E.A., Klimenko N.A., Problemy sovremennoj nauki i obrazovaniya, 2017, Vol. 87, No 5, pp. 7-13.

19. Suteu D., Coseri S., Rusu L., Desalination and Water Treatment, 2015, Vol. 57, No 31, pp. $14665-14673$

doi:10.1080/19443994.2015.1063464

20. Yousef R., Malika C., Water Sci Technol., 2020, Vol. 82, No 11. pp. 2366-2380. doi: $10.2166 /$ wst.2020.505

Voronyuk Iraida V. - Assistant professor, Department of Analytical Chemistry, Voronezh State University, Voronezh, e-mail: i.voronyuk@yandex.ru

Eliseeva Tatiana V. - Head of Department of Analytical Chemistry, Voronezh State University, Voronezh, e-mail: tatyanaeliseeva@yandex.ru

Selemenev Vladimir F. - DSc in chemistry, Voronezh State University, Voronezh, Russian Federation, e-mail: common@chem.vsu.ru 